\title{
A NEW COLOR CORRECTION METHOD FOR UNDERWATER IMAGING
}

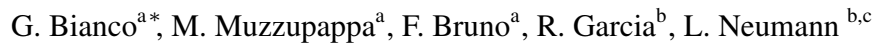 \\ a DIMEG, University of Calabria, 87036 Rende (CS), Italy - (gianfranco.bianco, muzzupappa, f.bruno)@unical.it \\ ${ }^{\mathrm{b}}$ Computer Vision and Robotics Group, University of Girona, 17001, Spain - rafael.garcia@udg.edu \\ ${ }^{\mathrm{c}}$ ICREA, Barcelona, Spain - Ineumann@ silver.udg.edu
}

\section{Commission V}

KEY WORDS: Underwater Imaging, Color Correction, Ruderman space, Computational Color Constancy

\begin{abstract}
:
Recovering correct or at least realistic colors of underwater scenes is a very challenging issue for imaging techniques, since illumination conditions in a refractive and turbid medium as the sea are seriously altered. The need to correct colors of underwater images or videos is an important task required in all image-based applications like 3D imaging, navigation, documentation, etc. Many imaging enhancement methods have been proposed in literature for these purposes. The advantage of these methods is that they do not require the knowledge of the medium physical parameters while some image adjustments can be performed manually (as histogram stretching) or automatically by algorithms based on some criteria as suggested from computational color constancy methods. One of the most popular criterion is based on gray-world hypothesis, which assumes that the average of the captured image should be gray. An interesting application of this assumption is performed in the Ruderman opponent color space $l \alpha \beta$, used in a previous work for hue correction of images captured under colored light sources, which allows to separate the luminance component of the scene from its chromatic components. In this work, we present the first proposal for color correction of underwater images by using $l \alpha \beta$ color space. In particular, the chromatic components are changed moving their distributions around the white point (white balancing) and histogram cutoff and stretching of the luminance component is performed to improve image contrast. The experimental results demonstrate the effectiveness of this method under gray-world assumption and supposing uniform illumination of the scene. Moreover, due to its low computational cost it is suitable for real-time implementation.
\end{abstract}

\section{INTRODUCTION}

Recovering correct or at least realistic colors of underwater scenes is a very challenging issue for the imaging techniques since illumination conditions in a refractive and turbid medium as the sea are seriously altered. Progressive attenuation with the depth of the visible spectrum at the different wavelengths, scattering and absorption of the incoming radiation from the ambient can cause relevant modifications of image colors (Schettini and Corchs, 2010). The need to correct colors of underwater images or videos is an important task required in all image-based applications like 3D imaging, navigation, image segmentation, documentation, etc. For dissemination purposes, it would be desirable to show 3D models of archaeological sites as represented on the land, or to improve the vision during underwater exploration and navigation, for example (Mahiddine et al., 2012; Gallo et al., 2012). The use of imaging devices with special optical filters or polarizers in front of the lens (Schechner and Averbuch, 2007) can attenuate part of the incoming radiation and often alter the real colors of the scene to be acquired. Moreover, the use of artificial light sources can introduce more non-uniformities in the lighting of the scene (Garcia et al., 2002). Many imaging techniques have been proposed in the literature for this task (Schettini and Corchs, 2010), based on a physical model of the image formation process (image restoration techniques) or qualitative, subjective criteria to produce a more realistic image (image enhancement methods). In the first case the knowledge of the medium physical parameters is required while in the other case some image adjustments can be performed manually (as histogram stretching) or based on some criteria as suggested from computational color constancy methods (Gijsenij et al., 2011). One of the most popular criteria is based on the gray-world hy-

\footnotetext{
${ }^{*}$ Corresponding author
}

pothesis, which assumes that the average of the captured image should be gray (Buchsbaum, 1980). This assumption allows to implement automatic methods which achieve good results. In (Reinhard et al., 2001) an interesting application of this assumption has been presented, by performing hue correction of images captured under colored light sources in the Ruderman opponent color space $l \alpha \beta$ (Ruderman et al., 1998). This color space, proposed to study the human eye perception of natural images, is composed of a luminance component $l$ (achromatic) and two opponent color components $\alpha$ and $\beta$, which encode yellow-blue and red-green chromaticities, as used for color discrimination in the human color visual mechanism. Until now, $l \alpha \beta$ space has been only used for color transfer applications (Reinhard et al., 2001), therefore, its ability to correct images taken under colored lighting is not yet deeply investigated, especially in underwater imaging.

In this work we present the first proposal for color correction of underwater images by using the $l \alpha \beta$ color space. In particular, the chromatic components are changed moving their distributions around the white point (white balancing) and histogram cutoff and stretching of the luminance component is performed to improve image contrast. Our main goal is to demonstrate the effectiveness of the color correction in this space for underwater scenes. Thus the improvement of image contrast and noise reduction are not investigated since we retain that such techniques are widely studied and proposed in literature, however they can be applied independently. The experimental results, presented below in this paper, demonstrate the effectiveness of this method under the hypothesis of gray world and uniform illumination of the scene. These assumptions are acceptable in many real cases for close-range acquisition in downward direction, like seafloor mapping, or capturing objects in the foreground (underwater photography) or less-complicated scenes where there are not strong light 
variations. As example of a possible application, we have processed an image set of an underwater finding with our method in order to obtain the 3D reconstruction by using a structure-frommotion technique. The proposed method requires just a single image and does not need any particular filters or prior knowledge of the scene. Moreover, due to its low computational cost it is suitable for real-time implementation.

The remaining of paper is organized as follow: in section 2 the main image enhancement methods for underwater imaging are summarized. To follow, the color alteration problem in underwater environments is described in section 3 and a few details as background are presented in section 4 . The color correction method is proposed in section 5 and the experimental results are reported in section 6. Finally, conclusions and future works are discussed in section 7 .

\section{RELATED WORK}

In this section the most important works about color correction of underwater images are presented. We focus on color correction strategies and methods used in the image enhancement techniques, which work on a single image without any knowledge about physical parameters or medium properties (image restoration methods). Essentially the different methods are composed by two steps: contrast enhancement and color correction, performed separately on RGB space or another one. From the analysis of the State of the Art, it is apparent that do not exist calibrated datasets of underwater images in order to compare and evaluate quantitatively the different methods from the point of view of computational color constancy (Gijsenij et al., 2011). In fact, it is difficult to obtain the ground truth, that is, a reference image taken with standard illumination to compare the results. Hence, the performance is evaluated qualitatively by visual inspection. For a more comprehensive and exhaustive treatise of the methods applied to improve quality of underwater images we suggest to refer to (Schettini and Corchs, 2010).

A histogram equalization method in RGB space is presented in (Shamsuddin et al., 2012). Color correction is carried out through both auto level adjustment and manual adjustment techniques. Hence, the authors found that the manual enhanced technique (done iteratively) is more precise as compared to the auto enhanced technique due to the levels of significance of $1 \%$ and $5 \%$. The images reported in the paper were taken in the shallow water (at depth from $1 \mathrm{~m}$ to $5 \mathrm{~m}$ ) in a coastal area of Malaysia. Iqbal et al. (2007) proposed an approach based on slide stretching. Firstly, contrast stretching of RGB algorithm to equalize the color contrast in the images is used. Secondly, the saturation and intensity stretching of HSV (Hue, Saturation, Value) is applied in order to increase the true colors and solve the lighting problem. The advantage of applying two stretching models is that it helps to equalize the color contrast in the images and also addresses the problem of lighting. The results are illustrated with images of fishes and marine flora captured in the foreground.

The HSV color space has been also used more recently in a twostage method (Ghani et al., 2014), for enhancing of coral images. First, the contrast correction technique is applied to the image with a modified Von Kries hypothesis and stretching it into two different intensity images at the average value with respect to Rayleigh distribution. At the second stage, color correction is performed in the HSV color model by stretching Saturation and Value (brightness) components. The stretching processes are applied to the histograms of both Saturation and Value at $1 \%$ to $99 \%$, respectively.

Bazeille et al. (2006) proposed an automatic algorithm to pre- process underwater images. It involves several successive independent processing steps which correct non-uniform illumination, suppress noise, enhance contrast and adjust colors. In particular, color correction is achieved by equalizing the mean of each color-channel. A linear translation of the histogram (for each RGB channel) is performed by adding to each pixel the difference between the desired mean value and the mean of the channel. Images taken under different conditions are showed and elaborated. A different type of equalization is proposed in (Chambah et al., 2003), by using Automatic Color Equalization (ACE), for automatic live fish recognition. This method merges two computational color constancy principles: gray world and retinex white patch. Lightness constancy makes stably perceive the scene regardless changes in mean luminance intensity and color constancy makes stably perceive the scene regardless changes in color of the illuminant. ACE is able to adapt to widely varying lighting conditions.

An approach based on the fusion principle has been presented by Ancuti et al. (2012) for enhancement of images and videos acquired in different lighting conditions. Two inputs are represented by color corrected and contrast enhanced versions of the original underwater image and also four weight maps that aim to increase the visibility. Color correction is performed by a white balancing process able to remove the color casts but also to recover the white and gray shades of the image, based on the grayworld assumption. In particular, the authors propose to increase the average value estimated with a percentage $\lambda \in[0,0.5]$.

A very different approach has been used by Petit et al. (2009), which propose a method based on light attenuation inversion after processing a color space contraction using quaternions. Applied to the white, the attenuation gives a hue vector characterizing the water color. Using this reference axis, geometrical transformations into the color space are computed with quaternions. Pixels of water areas of processed images are moved to gray or colors with a low saturation whereas the objects remain fully colored. Thus, the contrast of the observed scene is significantly improved and the difference between the background and the rest of the image is increased. Moreover, Principal Component Analysis (PCA) calculation allows to extract a dominant color of the image, hence, in most cases, the water color provides also good results in term of color enhancement by using the method described above. The results are showed on images with a large presence of a water area.

In (Gouinaud et al., 2011) the authors introduce a Color Logarithmic Image Processing (CoLIP) framework. This novel framework expands the logarithmic image processing theory to color images in the context of the human color visual perception. The color correction performs a CoLIP subtraction of the color in the image that should be white to the eye under daylight illumination, that is, a pixel of the image that is recognized as white under standard illumination. From the LMS space expressed following the LIP approach, a three-component function is defined (color tone function). Its first component is the achromatic tone function, and the last components are the two chromatic antagonist tone functions. This method has been successfully evaluated on both underwater images (blue illuminant) and indoor reflected light images (yellow illuminant).

Color recovery is also analysed by Torres-Mendez and Dudek (2005) but from a different perspective: it is formulated as an energy minimization problem using learned constraints. An image is modeled as a sample function of Markov Random Field and the color correction is considered as a task of assigning a color value to each pixel of the input image that best describes its surrounding structure using the training image patches. Training images are small patches of regions of interest that capture the maximum of the intensity variations from the image to be restored. The im- 
ages proposed as result regard fishes in foreground or scene in shallow water. To evaluate the performance of the algorithm the authors use a dataset (available on a website) of underwater images taken with a professional camera and in most of the cases they were also enhanced by using a commercial software. They consider these dataset images as ground truth and apply an attenuation filter to obtain a color depleted image. As metric the mean absolute residual error in CIELab space is computed between the ground truth and the color corrected images.

Also dehazing techniques have been applied in underwater imaging. Chiang and Chen (2012) propose a novel dehazing algorithm for wavelength compensation and image dehazing in order to remove distortions caused by light scattering and color change, respectively. Energy compensation for each color channel is carried out after water depth estimation to adjust the bluish tone to a natural color. The algorithm performance is evaluated by utilizing ground-truth color patches and video available on internet. In particular, a board with six color patches is captured in the close range before diving (ground truth) and at a depth of 5 and $15 \mathrm{~m}$, and as measurement of the error the authors report the SNR (Signal-to-Noise) ratio. In (Carlevaris-Bianco et al., 2010) a dehazing algorithm for removing the effects of light scattering in underwater images is presented. The underwater model takes into account the absorption as function of both scene depth and wavelength, which attenuating the red color channel much more severely than blue or green. Color correction is provided for the reduction in the red channel caused by absorption. Moreover the estimation of the airlight (blue in water) includes the effects of absorption on the airlight. Therefore, when the additive airlight has removed, the color balance of the imagery is improved, without having to additionally account for absorption effects. In order to test the color accuracy, multiple images of a color wheel target are collected at varying distances between 3 and $6 \mathrm{~m}$ from the camera in a fresh water test tank. Then, an image of the color wheel taken in air is used as the ground truth.

\section{UNDERWATER COLOR ALTERATIONS}

The sunlight that penetrates from the air-water interface into the water is altered from several causes. Firstly, the visible spectrum is modified with the depth because of the radiation absorption at the different wavelengths, in particular, the radiations with lower frequency are more absorbed. Thus, the red components disappear already within about $5 \mathrm{~m}$ (shallow waters), the orange at 7.5 $\mathrm{m}$, the yellow in the range of 10-14 m, and the green at about 21 $\mathrm{m}$. A schematic representation of this color loss is illustrated in Figure 1. It is evident that more depth increases, more the lower temperature colors disappear, producing greenish-blue scenes. It needs also to take into account the scattering (backward and forward) and absorption effects due to the particles dissolved and suspended in the medium, like salt, clay and micro-organisms. The greater the dissolved and suspended matter, the greener (or browner) the water becomes. These particles not only alter the colors but also attenuate and diffuse more the light with consequent decreasing of contrast and increasing of blur and noise in the captured images. Let us remember that when we capture horizontally an underwater image, we have to consider as the light path the horizontal distance from the target (working distance) and the depth which we are, hence, the radiation suffers twice the absorption. As depicted in Figure 1 light intensity that penetrates into the water already decays of $50 \%$ at $10 \mathrm{~m}$. Moreover, under water the color light depends on other factors as the coastal proximity, the season, the water surface conditions, the time of the day, the cloudiness of the sky, the presence of marine vegetation or the soil type. For the reasons listed above, it is clear that underwater environments offer a non-constant and multi-variate typology of light conditions. Since many of the aforementioned factors are constantly changing, we cannot really know all the color variations in water.

sunlight

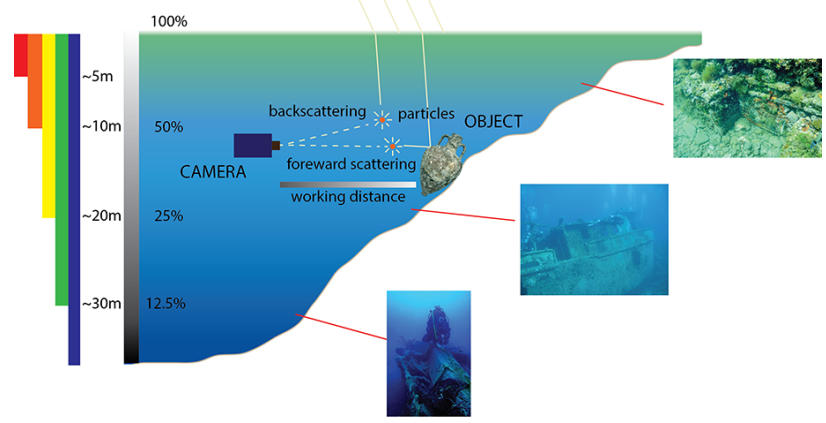

Figure 1: Schematic representation of underwater lighting conditions that can cause color alterations.

\section{BACKGROUND}

Before to describe the proposed color correction method, we provide some details about color constancy, white balancing, image formation model and gray world assumption. The $l \alpha \beta$ color space is also introduced.

\subsection{Formulation of the color correction problem}

Recognizing correct casts of objects in a scene illuminated by a colored light source is a capability of the human visual system, called color constancy (Ebner, 2007). In fact, the human visual system can perceive the true colors of objects even if not illuminated by a neutral light source, i.e. white. For example, a white object illuminated with a high temperature light source becomes bluish, but the human observer will still recognize it as white. On the other hand, for digital systems as imaging devices, it is necessary to adjust color casts after acquisition, in order to recover the correct colors, because sensors measure the incoming light from the objects, and they are not able to distinguish between the color of the illuminant and the reflectance of the objects. In digital photography, the process (automatic or manual) to remove unrealistic color casts is called white balancing, which allows to obtain a balanced color distribution around a white point taken as reference. Digital cameras are able to balance color images under a wide variety of illumination conditions (i.e. neon light, sun light, cloudy sky) by internal processes but these settings often fall in underwater environments. For a more accurate correction a calibration chart or a color checker can be placed and captured in the scene. Then in post-processing the images are corrected. The use of these calibrated charts underwater is not suitable and practical for applications like mapping and navigation, in which a large amount of data is collected.

In this work we tackle the color correction problem of underwater images from the point of view of computational color constancy, which aims to perform a color correction that closely mimics the performance of the visual system. We suppose that the scene is illuminated by a colored light source uniformly. This means that the light intensity is approximately constant over whole image. Moreover we not consider degradation effects which cause noise and blur in the image due to scattering and we suppose 
that the object surfaces are Lambertian, that means the surface reflects the light equally in all directions. These assumptions can be considered too restrictive, but allow to use a simply image formation model, and however, they are acceptable in many real cases for close-range acquisition in downward direction, like seafloor mapping, or capturing objects in the foreground or lesscomplicated scenes where there are not strong light variations. Thus, we can formulate the problem in a simplified manner following the computational color constancy approach. In the image formation model (Gonzales and Woods, 1992), as represented in Figure 2, we can consider an underwater image $f_{i}(m, n)$ expressed by:

$$
f_{i}(m, n)=g_{i}(m, n) \cdot r_{i}(m, n)
$$

where

$$
\begin{aligned}
& g_{i}(m, n)=\text { illuminant } \\
& r_{i}(m, n)=\text { reflectance } \\
& (m, n)=\text { image coordinates }
\end{aligned}
$$

with $i \in\{1,2,3\}$ we indicate the three color components of image, corresponding in RGB space to $\{R, G, B\}$ channels. Correcting the color casts of an image means computing the illuminant $g_{i}$ and removing its chromatic components from $f_{i}$. In order to compute the color of illuminant further assumptions are needed and for this purpose several computational color constancy methods have been proposed in literature (Gijsenij et al., 2011). In the next section we describe the formulation of the gray-world assumption used in our method.

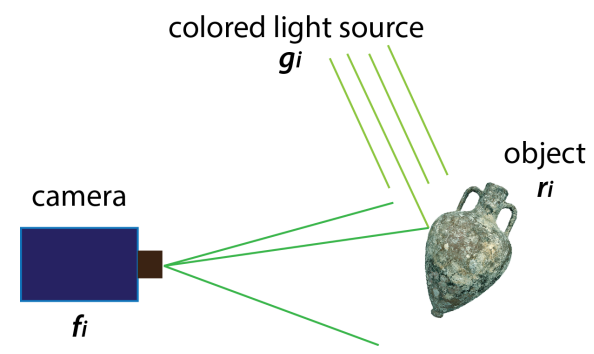

Figure 2: Underwater reflectance model.

\subsection{The gray-world assumption}

The gray-world assumption is a well-known approach, proposed by Buchsbaum (Buchsbaum, 1980). In its simplest version it assumes that the information in the average of each channel of the image is the representative gray level. In other words, the average of the captured image should be gray (achromatic). Following the image formation model described above in Eq. 1 and taking into account that the illuminant $g_{i}$ can be approximate (Ebner, 2007) by the average of image $f_{i}$, the gray world assumption can be formulated as:

$$
f_{i}^{*}(m, n)=\frac{f_{i}(m, n)}{g_{i}(m, n)} \approx \frac{f_{i}(m, n)}{\bar{f}_{i}(m, n)}
$$

$i \in\{1,2,3\}$ is the number of channels, corresponding in RGB space to $\{R, G, B\}$ and $\bar{f}_{i}=\operatorname{mean}\left\{f_{i}(m, n)\right\}$. The main drawback of gray-world methods is that they will fail to produce correct colors if sufficiently large numbers of hues are not present in the scene.

\section{3 l $l \alpha \beta$ color space}

The proposed method aims to perform a color correction that closely mimics the performance of the visual system. For this reason we have chosen to adopt a perceptual color space as we described below. The $l \alpha \beta$ color space has been proposed by Ruderman et al. (1998) studying the human eye perception of natural images. It is composed of a luminance component $l$ (achromatic) and two opponent color components $\alpha$ and $\beta$, as in the human color visual mechanism. Until now this color space has been only used for color transfer applications. In particular, Reynard et al. (2001) have showed that it can be used for hue correction, presenting the results obtained to correct an image with uniform illumination, under red, tungsten, and blue illuminants. Therefore color correction by using this color space is not yet deeply investigated, especially in underwater imaging.

Here, we describe $l \alpha \beta$ space following the transformations matrices suggested in (Reynard et al., 2001), that we have reported in Appendix for the reader's convenience. The several steps to convert RGB coordinates in $l \alpha \beta$ space are reported in the flow-chart of Figure 3. First of all, RGB image has to be corrected by the non linearity (gamma correction), to work with linear RGB coordinates. This step is very important and necessary to pass to the next: conversion in the XYZ tristimulus values. This conversion is achieved by multiplying the linear RGB coordinates $f_{i}(m, n)$ with $T_{x y z, i j}$ matrix, where the $i$ and $j$ are the matrix indeces in according to the Einstein notation:

$$
x_{j}(m, n)=T_{x y z, i j} f_{i}(m, n)
$$

where $x_{j}$ is the vector of coordinates in XYZ space and $(m, n)$ are the pixel coordinates. From this device-independent XYZ space, we convert the image to LMS space $\left(l_{j}(m, n)\right.$ vector) using the $T_{l m s, i j}$ matrix:

$$
l_{j}(m, n)=T_{l m s, i j} x_{i}(m, n)
$$

Now, we transform the data in logarithmic space:

$$
l_{\log , j}(m, n)=\log l_{i}(m, n)
$$

and finally we can proceed to decorrelate these axes as suggested by Ruderman et al. (1998), by using Principal Components Analysis (PCA), that means multiply $l_{\log , j}(m, n)$ vector by the decorrelation matrix $T_{p c a, i j}$ :

$$
l_{l \alpha \beta, j}(m, n)=T_{p c a, i j} l_{l o g, i}(m, n)
$$

Thus, we have obtained the vector coordinates $l_{l \alpha \beta, j}(m, n)$ of image in $l \alpha \beta$ space. The three resulting principal axes are orthogonal, and it is found that the first axis $l$ represents an achromatic channel, while the second $(\alpha)$ and the third $(\beta)$ channels are chromatic yellow-blue and red-green opponent channels:

$$
\begin{aligned}
& \text { - luminance : Achromatic } \propto(r+g+b) \\
& \text { - } \alpha \text { : Yellow-blue } \propto(r+g-b) \\
& \text { - } \beta \text { : Red-green } \propto(r-g)
\end{aligned}
$$

Hence, in this space the color components are decoupled from the luminance, that is, the brightness level of the scene. The $l \alpha \beta$ is a perceptual color space, that is, based on the response functions of the cones in the retina of the eye, because it is derived by the perceptual color space LMS (Long, Medium, Short) mostly used in psychometric research.

\section{COLOR CORRECTION METHOD}

In this section we describe the color correction method that we propose for underwater imaging. The method is based on the 


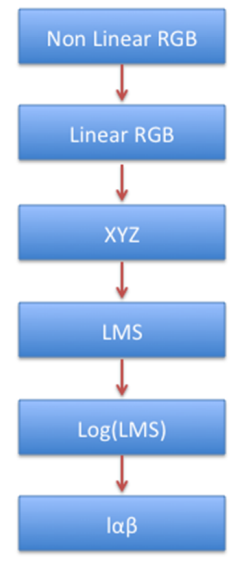

Figure 3: Flow-chart: conversion of a non linear RGB image in $1 \alpha \beta$ space.

assumptions of gray world and uniform illumination of the scene, as explained in the previous section. Thus, we formulate the Eq. 2 in $l \alpha \beta$ space. Let us express the RGB color coordinates $f_{i}(m, n)$ in the LMS space $\left(l_{i}(m, n)\right)$ as:

$$
l_{i}(m, n)=D_{i j} \cdot f_{j}(m, n)
$$

where $D_{i j}$ is the transformation matrix obtained as product of the two transformation matrices $D_{i j}=T_{x y z, i j} \cdot T_{l m s, i j}$. Expressing the terms of Eq. 2 in LMS space in according to Eq. 7 and computing the logarithm of both sides of the Eq. 2, we have:

$$
\log l_{i}^{*}(m, n)=\log \frac{l_{i}(m, n)}{\overline{l_{i}}}
$$

where $l_{l m s, i}^{*}(m, n)$ are the corrected coordinates in LMS space of $f_{i}^{*}(m, n), l_{l m s, i}(m, n)$ are the coordinates of $f_{i}(m, n)$ and $\overline{l_{i}}$ its average in according to gray-world assumption. Applying the logarithm quotient rule and multiplying by the $T_{p c a, i j}$ matrix, we obtain:

$$
T_{p c a, i j} \log l_{i}^{*}(m, n)=T_{p c a, i j} \log l_{i}(m, n)-T_{p c a, i j} \log \bar{l}_{i} ;
$$

Each term in this equation is similar to Eq. 6, that states the coordinates conversion in the $l \alpha \beta$ space. Thus, we have obtained the desired expression of Eq. 2 in $l \alpha \beta$ coordinates:

$$
l_{l \alpha \beta, i}^{*}(m, n)=l_{l \alpha \beta, i}(m, n)-\bar{l}_{l \alpha \beta, i} ;
$$

where $l_{l_{\alpha \beta, i}}^{*}(m, n)$ are the corrected coordinates, $l_{l \alpha \beta, i}(m, n)$ the component of RGB image and $\bar{l}_{l \alpha \beta, i}$ its average. In order to correct color casts of an image the $\alpha$ and $\beta$ components $(i \in$ $\{2,3\}$ ) must be changed, as suggested in (Reynard et al., 2001), while the luminance component remains unchanged, at least regarding the translation of the mean, but it will be elaborated in a different way, to improve the contrast image, as described in the next section. After color correction processing we must transfer the result back to RGB to display it by applying the inverse operations. It has to be noted that the white point $(\{1,1,1\}$ in RGB space) has $\{0,0,0\}$ coordinates in $l \alpha \beta$ space. This means that the method performs a shift of $\alpha$ and $\beta$ components respect to the coordinates $\{0,0\}$, putting the distributions of these coordinates around the white point. Correcting image casts respect to the white point in this space seems to be more realistic respect to adjust the distributions of color channels around a gray point as in RGB space. We can conclude that the gray-world assumption is transformed in a "white-world" assumption in the $l \alpha \beta$ space.

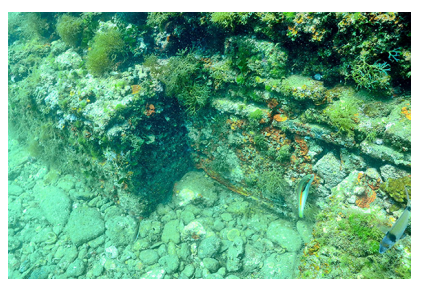

(a)

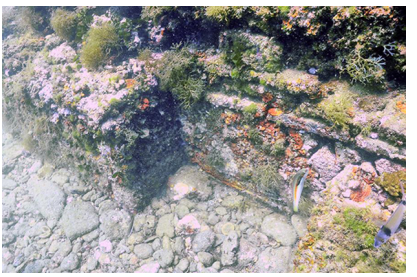

(c)

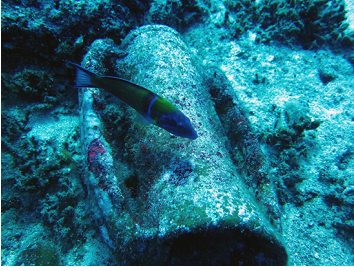

(b)

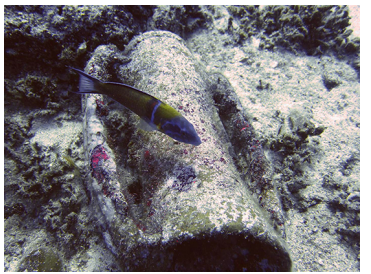

(d)
Figure 4: Two cases of underwater images analysed in this work (a), (b), and the relative results obtained with our algorithm (c), (d).

\section{EXPERIMENTAL RESULTS}

In order to demonstrate the effectiveness of the proposed method, we have tested it on two underwater images captured in different locations (Figures 4a,b). The first case that we analysed is an image corresponding to a part of a brick wall, taken in the archaeological site of Baia (Naples-Italy) at the depth of about $5 \mathrm{~m}$, in which a greenish component is present. The second case is an image representing an amphora (Kas, Turkey) in which a bluish component is dominant. This image is taken from a website of divers community (Worldadventuredivers, 2015).

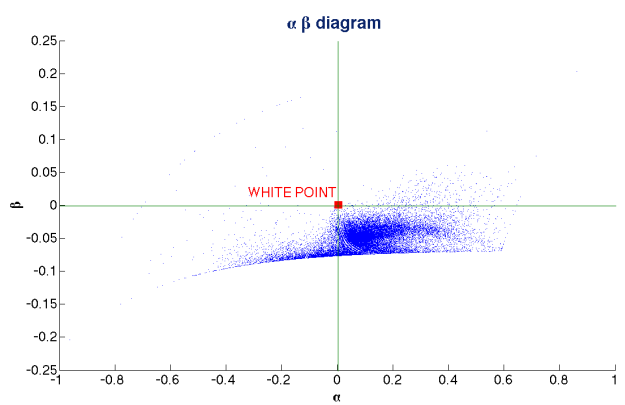

(a)

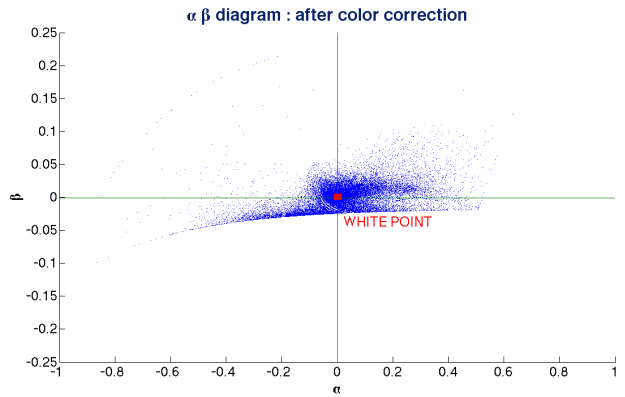

(b)

Figure 5: Distribution of chromatic components of image in Figure $4 \mathrm{a}$ in $\alpha-\beta$ plane before and after color correction.

The two RGB images are converted in the $l \alpha \beta$ space, following the steps discussed in section 4.3. Then we apply the Eq. 10 to $\alpha$ 


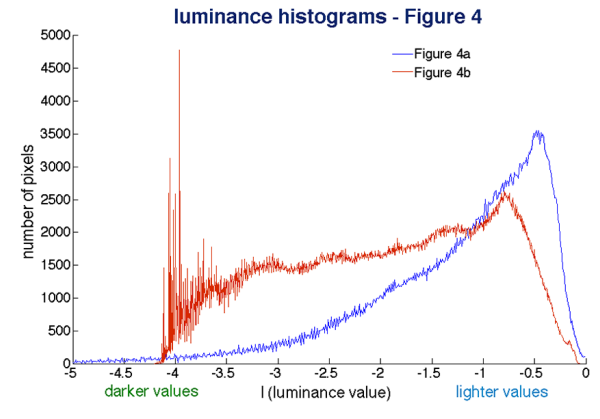

Figure 6: Luminance histograms of images in Figure 4.
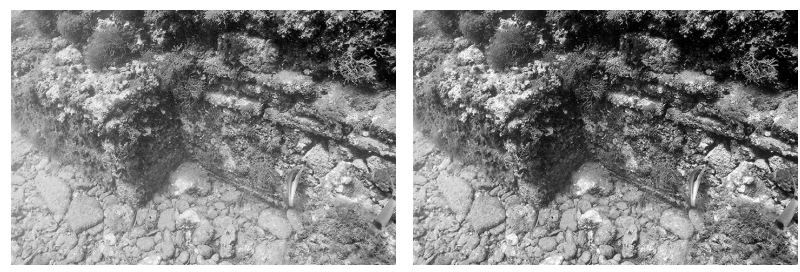

Figure 7: Image of the luminance channel before (at left) and after (at right) contrast enhancement by histogram stretching.

and $\beta$ components. According to our experience, it is preferred for underwater images to use channel-wise median values instead of the mean, since the median is not affected by extreme values in luminance or chrominance. In this way the median would ignore, for instance, overlighted close objects or the blue parts of the image corresponding to farther distances, which, on the other hand, would affect the mean. As example of the result of the white balancing process, we plot in Figure 5 in the $\alpha-\beta$ plane the distribution of the chromatic components for the image of Figure 4a. It is apparent that after color correction, the distribution of $\alpha$ and $\beta$ is centered on the white point coordinates $\{0$, $0\}$. Moreover, the $\beta$ channel is more affected by the illumination color, while $\alpha$ channel remains approximately unchanged. In fact, we have found that for underwater images the median value of $\beta$ is always negative. In order to verify the hypothesis of uniform illumination, we have computed the luminance histograms, of both images as depicted in Figure 6. The values of luminance distribution are sparse from darker values to the lighter values: the image histogram of Figure $4 \mathrm{a}$ has a bell-shape distribution concentrated on lighter values, while the one of Figure $4 \mathrm{~b}$ has a distribution more equalized. In case of the luminance values are concentrated around two or more picks, especially in the brighter area, the uniform-illumination hypothesis has to be rejected.

In Figure $7 \mathrm{a}$ the image of the luminance channel is illustrated. It has been obtained by setting to zero the chromatic components and reporting back the coordinates in RGB space. The result of this process is a gray-level image, as we expected because the luminance channel is achromatic. In order to improve the image contrast, in this work we propose to perform a cutoff of $1 \%$ of both histogram limits and stretching them. This is a simple way to improve the contrast image, but also other contrast enhancement techniques can be applied like CLAHE (Contrast Limited Adaptive Histogram Equalization) or histogram equalization, for example. The result of the luminance histogram stretching is illustrated in Figure 7. Finally, we combine the results of luminance enhancement and hue correction, then we apply the inverse transformations of the flow-chart in Figure 3 to report the results in RGB space, as shown in Figures 4c,d. Regarding computational considerations, we have implemented a MATLAB $R$ code

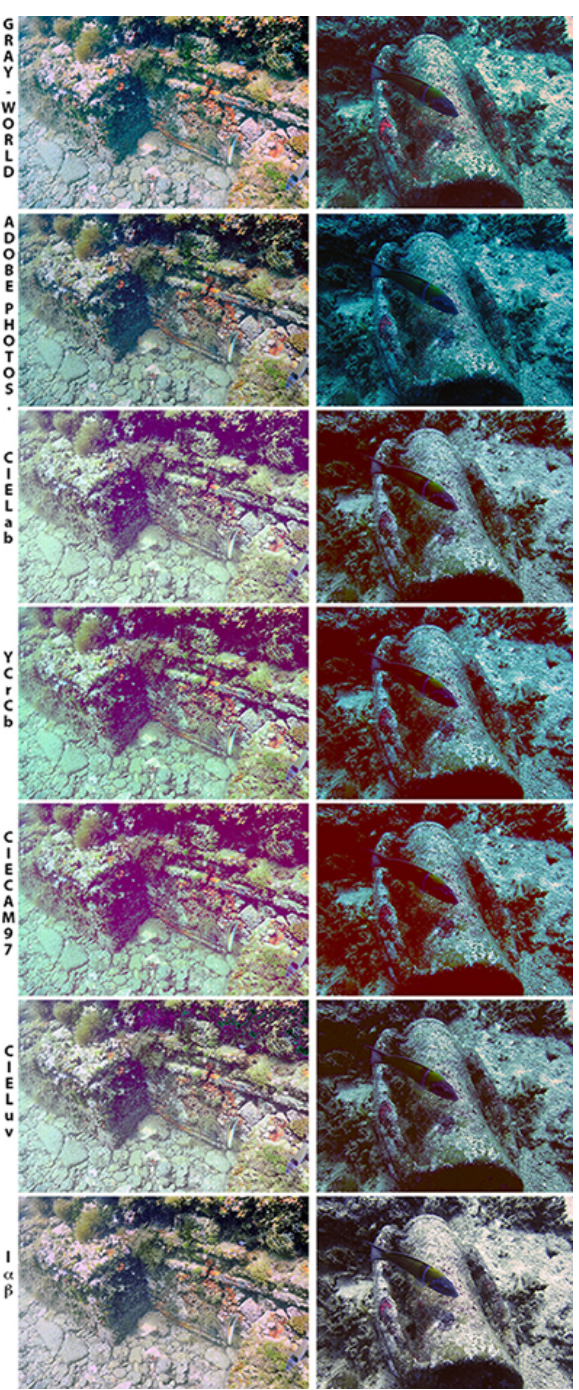

Figure 8: Comparison among different color correction methods.

to process underwater images with the proposed method. It takes $0.795 \mathrm{sec}$ on a Mac Laptop (Processor $2.7 \mathrm{GHz}$ Core i7, $16 \mathrm{~GB}$ 1600 MHz DDR3 Memory, NVIDIA GeForce GT 650M 1024 MB) to elaborate a $591 \times 892$ image. We point out that the implementation of this algorithm can be improved by using parallel computing or $\mathrm{C}$ implementation, thus we think that the method is suitable for real-time applications.

\subsection{Comparisons with other color spaces}

From the study of the State of the Art we found that underwater image datasets provided with calibrated ground truth are not available in literature as for the in-air applications of computational color constancy algorithms (Gijsenij et al., 2011). Hence we limit the performance evaluation of our method to a qualitative evaluation by visual inspection. We have compared the white balancing results of our method with the ones obtained with other similar color spaces, in order to investigate if the $l \alpha \beta$ space allows to give similar or better results. For this reason we have performed this analysis on CIELab, YCrCb, CIECAM97, CIELuv spaces, which are composed from a luminance component and two chromatic components like $l \alpha \beta$ space. Similarly to the white balancing done in $l \alpha \beta$ space, we have shifted the median values of chromatic components around its own white point. Moreover we have elaborated the underwater images of Figure 4 with a 
gray-world algorithm in RGB space (Colorconstancy, 2015) and with Photoshop software by using "Auto Color" tool. The outputs of each aforementioned methods are illustrated in Figure 8. From the comparison it is apparent that the $l \alpha \beta$ space gives the best results of color correction removing completely in the whole image the greenish and bluish casts. In the case of gray-world result, the correction seams globally acceptable and the colors are most saturated, but by a more accurate inspection we can see that in the darker and lighter areas (pebbles on the seafloor), remain colored casts. Color correction in $l \alpha \beta$ space outputs images with realistic color casts both in gray shades and bright areas.

\subsection{D reconstruction application}

As example of a possible application, we have processed an image set of an underwater finding with our method in order to obtain the 3D reconstruction by using a structure-from-motion software. The dataset has been taken in the archaeological site of Kaulon in Monasterace (Calabria - Italy), capturing a remainder of an ancient pillar. The dataset is composed by 45 images with resolution of $980 \times 1479$ pixels. We have pre-processed all the images with our method and then input in a structure-frommotion algorithm. The results of color correction and the 3D model obtained by the corrected dataset is shown in Figure 9. As an alternative we can process directly the texture applied on the 3D model and obtained by UV mapping.

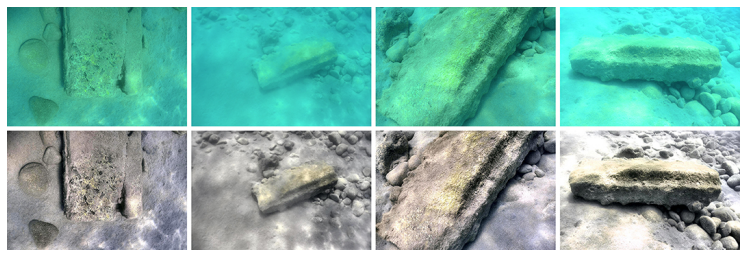

(a)

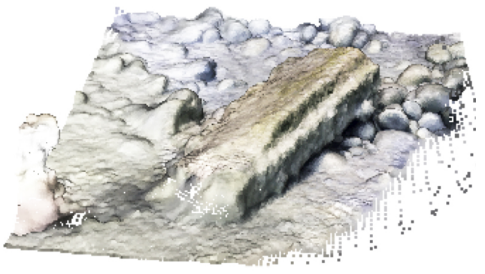

(b)

Figure 9: 3D model (b) obtained from a color corrected dataset of underwater images (a).

\section{CONCLUSIONS AND FUTURE WORKS}

We presented a new method for color correction of underwater images. The method is based on gray-world and uniform illumination assumptions applied in the $l \alpha \beta$ space. Color correction is performed by white balancing of chromatic components ( $\alpha$ and $\beta$ ), while the luminance component $l$ is processed to improve the image contrast. We have tackled the color correction problem with a computational color constancy approach, developing the mathematical formulation of the hue correction suggested by Reynhard et al. (2001). From the experimental results, we demonstrated that this method is suitable to remove undesired color casts in underwater imaging: greenish-blue components in the images are completely removed also in the gray shades. From the analysis of the State of the Art, we have found out that until now it does not exist a calibrated underwater dataset provided of ground truth, in order to perform quantitative evaluations of the methods proposed in literature. Thus, a qualitative comparison of white balancing process, in other perceptual color spaces, shows that the hue correction in $l \alpha \beta$ space outputs the best results, also respect to a RGB gray-world algorithm and a commercial software.

As example of a possible application, we have proposed to preprocess underwater image dataset in order to improve the results of structure-from-motion software, obtaining 3D models with color corrected texture. These promising results convince us to deepen the investigations of this method for underwater imaging. In particular, further works will regard the comparisons with the other methods presented in literature, studying an experimental protocol to acquire an appropriate dataset in order to evaluate quantitatively the error in the color correction, implement a new version of our method to take into account the non-uniform lighting conditions and apply better contrast enhancement techniques to reduce the degradation effects, like noise and blur. We will study instead average color an appropriately weighted median color, too.

\section{ACKNOWLEDGEMENTS}

This work has been supported by the project "ARUE" (MIDAVI 55), funded with support from the European Commission, European Social Fund and Regione Calabria. This publication reflects the views only of the author, and the European Commission and the Regione Calabria cannot be held responsible for any use which may be made of the information contained therein. Moreover, this work has been partially supported by the Project 'CoMAS' (ref. PON01_02140), financed by the MIUR under the PON "R\&C" 2007/2013 (D.D. Prot.n.01/Ric.18.1.2010).

\section{REFERENCES}

Ancuti, C., Ancuti, C. O., Haber, T., Bekaert, P., 2012. Enhancing underwater images and videos by fusion. In Computer Vision and Pattern Recognition (CVPR), IEEE Conference on, pp. 81-88.

Bazeille, S., Quidu, I., Jaulin, L., Malkasse, J.P., 2006. Automatic Underwater Image Pre-Processing. CMMO6 - CARACTERISATION DU MILIEU MARIN, 16-19 Oct.

Buchsbaum, G., 1980. A spatial processor model for object colour perception, Journal of The Franklin Institute-engineering and Applied Mathematics, 310:126.

Carlevaris-Bianco, N., Mohan, A., Eustice, R. M., 2010. Initial results in underwater single image dehazing. In OCEANS 2010 , pp. 1-8.

Chambah, M., Semani, D., Renouf, A., Courtellemont, P., Rizzi, A., 2003. Underwater color constancy: enhancement of automatic live fish recognition. In Electronic Imaging, International Society for Optics and Photonics, pp. 157-168.

Chiang, J. Y., Chen, Y. C., 2012. Underwater image enhancement by wavelength compensation and dehazing. Image Processing, IEEE Transactions on, 21(4), 1756-1769.

Colorconstancy, 2015. http://colorconstancy.com/, Last accessed on 2015, March 14.

Ebner., M., 2007. Color Constancy, Wiley 1st edition.

Gallo, A., Angilica, A., Bianco, G., De Filippo, F., Muzzupappa, M., Davidde, B., Bruno, F., 2012. 3D Reconstruction and Virtual 
Exploration of Submerged Structures: a Case Study in the Underwater Archaeological Site of Baia (Italy). In VAST: International Symposium on Virtual Reality, Archaeology and Intelligent Cultural Heritage, Eurographics Association, pp. 121-128.

Garcia, R., Nicosevici, T., Cufi, X., 2002. On the way to solve lighting problems in underwater imaging, In OCEANS '02 MTS / IEEE, pp.1018-1024 vol.2, 29-31 Oct.

Ghani, A. S. A., Isa, N. A. M., 2014. Underwater image quality enhancement through composition of dual-intensity images and Rayleigh-stretching. SpringerPlus, 3(1), 757.

Gijsenij, A., Gevers, T., van de Weijer, J., 2011. Computational Color Constancy: Survey and Experiments, Image Processing, IEEE Transactions on, vol.20, no.9, pp.2475-2489.

Gonzalez, R.C., Woods, R.E., 1992. Digital Image Processing. Addison-Wesley, Reading, MA.

Gouinaud, H., Gavet, Y., Debayle, J., Pinoli, J. C., 2011. Color correction in the framework of color logarithmic image processing. In Image and Signal Processing and Analysis (ISPA), 7th International Symposium on, pp. 129-133.

Iqbal, K., Abdul Salam, R., Osman, M., Talib, A. Z., 2007. Underwater Image Enhancement Using An Integrated Colour Model. IAENG International Journal of Computer Science, 32(2), 239244.

Mahiddine, A., Seinturier, J., Boi, D.P.J., Drap, P., Merad, D., Luc Long, 2012. Underwater image preprocessing for automated photogrammetry in high turbidity water: An application on the Arles-Rhone XIII roman wreck in the Rhodano river, France, In Virtual Systems and Multimedia (VSMM), 2012 18th International Conference on, pp.189,194, 2-5 Sept.

Petit, F., Capelle-Laize, A., Carr, P., 2009. Underwater image enhancement by attenuation inversionwith quaternions. In Acoustics, Speech and Signal Processing, IEEE International Conference on, pp. 1177-1180.

Reinhard, E., Ashikhmin, M., Gooch, B.,Shirley, P., 2001. Color Transfer between Images, IEEE Computer Graphics and Applications, vol. 21, no. 5, pp. 34-41.

Ruderman, D., Cronin, T., Chiao, C., 1998. Statistics of cone responses to natural images: implications for visual coding, $J$. Opt. Soc. Am. A 15, 2036-2045.

Schechner, Y., Averbuch, Y., 2007. Regularized image recovery in scattering media. IEEE Trans Pattern Anal Mach Intell.

Schettini, R., Corchs, S., 2010. Underwater image processing: state of the art of restoration and image enhancement methods. EURASIP Journal on Advances in Signal Processing 2010, 14.

Shamsuddin, N. B., Fatimah, W., Ahmad, W., Baharudin, B. B., Kushairi, M., Rajuddin, M., Mohd, F. B., 2012. Significance Level of Image Enhancement Techniques for Underwater Images. In IVIC 2012.

Torres-Mndez, L. A., Dudek, G., 2005. Color correction of underwater images for aquatic robot inspection. In Energy Minimization Methods in Computer Vision and Pattern Recognition pp. 60-73, Springer Berlin Heidelberg.

Worldadventuredivers, 2015. http://worldadventuredivers.com/, Last accessed on 2015, March 14.

\section{APPENDIX}

We report the conversion matrices used by Reinard et al. (2001).
- Conversion from linear RGB to XYZ space:

$$
\begin{gathered}
x_{j}(m, n)=T_{x y z, i j} f_{i}(m, n) \\
T_{x y z, i j}=\left[\begin{array}{lll}
0.5141 & 0.3239 & 0.1604 \\
0.2651 & 0.6702 & 0.0641 \\
0.0241 & 0.1228 & 0.8444
\end{array}\right]
\end{gathered}
$$

- Conversion from XYZ space to LMS:

$$
\begin{gathered}
l_{j}(m, n)=T_{l m s, i j} x_{i}(m, n) \\
T_{l m s, i j}=\left[\begin{array}{ccc}
0.3897 & 0.6890 & 0.0787 \\
-0.2298 & 1.1834 & 0.0464 \\
0.0000 & 0.0000 & 1.0000
\end{array}\right]
\end{gathered}
$$

- Conversion from $\log (\mathrm{LMS})$ space to $l \alpha \beta$ space:

$$
\begin{gathered}
l_{l \alpha \beta, j}(m, n)=T_{p c a, i j} l_{l o g, i}(m, n) \\
T_{p c a, i j}=\left[\begin{array}{ccc}
\frac{1}{\sqrt{3}} & 0 & 0 \\
0 & \frac{1}{\sqrt{6}} & 0 \\
0 & 0 & \frac{1}{\sqrt{2}}
\end{array}\right]\left[\begin{array}{ccc}
1 & 1 & 1 \\
1 & 1 & -2 \\
1 & -1 & 0
\end{array}\right]
\end{gathered}
$$

